\title{
O ENSINO TEÓRICO-PRÁTICO DA ASSISTÊNCIA DE ENFERMAGEM NUMA INSTITUIÇÃO DE ENSINO - UM ESTUDO DE REFLEXÃO*
}

\author{
Bertha Cruz Enders** \\ Maria Élida Santos de Souza*** \\ Jacinta Maria Morais Formiga**** \\ Rejane Maria Paiva de Menezes*****
}

\begin{abstract}
RESUMO - O trabalho é um análise de ensino teórico-prático da assistência de enfermagem no curso de graduação da Universidade Federal do Rio Grande do Norte objetivando identificar 1) os elementos do conceito da assistência de enfermagem do curso, 2) os métodos de ensino da assistência e 3) os padrões de avaliação utilizados. A análise foi realizada com base nas reflexōes de docentes representantes das disciplinas com conteúdo teóricoprático de assistência. Identificaram-se 1) desarticulações internas quanto aos conceitos, 2) desvinculação do ensino e prática da assistência nos serviços, e 3) falta de padrões de qualidade para avaliação da assistência, problemas estes oriundos da desintegração interdisciplinar e do ensino-serviço. Propõe-se uma colaboração docente-assistencial na definição conceitual, teórica e prática da assistência de enfermagem.
\end{abstract}

\begin{abstract}
The study is an analysis of theory and practicum teaching of nursing care in the undergraduate program of the Federal University of Rio Grande do Norte. The objectives were to identify 1) the conceptual elements of nursing care in the program, 2) the teaching methods used, and 3) the criteria for quality evaluation of care. Professors who taught theory and practicum of nursing analysed their considerations related to the teaching and practice of nursing care expressed at discussion meetings. The results indicated a lack of internal integration, and between teaching and service characterized by 1) disarticulation of concepts, 2) lack of coherence between teaching and service and 3) lack of parameters, for the evaluation of care. Suggestions are made for collaboration of teaching and service professionals in defining their conceptual, theoretical, and practicum bases for care.
\end{abstract}

\section{INTRODUÇÃO}

Nas últimas décadas, a qualidade da assistência à saúde da população tem decaído consideravelmente. A enfermagem, como as demais prof issões com função social e que responde por parte significante dessa assistência, vem se preocupando sensivelmente na busca coletiva de soluções para os problemas de qualificação da assistência prestada.

Outrossim, a necessidade dos profissionais de enfermagem em parar e refletir sobre a assistência prestada é de suma importância no momento em que se percebe uma perspectiva de reforma e de qualificação na área da saúde em todo o país. Cabe aos enfermeiros assistenciais e docentes tal empreendimento.

O propósito deste trabalho é analisar o ensino teórico-prático da assistência de enfermagem no curso de graduação da Universidade Federal do Rio Grande do Norte (UFRN).

O trabalho é o resultado de um estudo de reflexões realizado por um grupo de enfermeiras docentes representantes das disciplinas que possuem conteúdo de ensino teórico-prático da assistência de enfermagem.

* Trabalho classificado em $1^{\circ}$ lugar-Prêmio Wanda Horta-41-CBEn-Florianópolis.

** Enfermeira - Doutora em Enfermagem (Ph.D) - Professor Adjunto do Departamento de Enfermagem da Universidade Federal do Rio Grande do Norte.

*** Enfermeira - Mestre em Enfermagem - Professor Adjunto do Departamento de Enfermagem da Universidade Federal do Rio Grande do Norte.

**** Enfermeira-Professor Assistente do Departamento de Enfermagem da Universidade Federal do Rio Grande do Norte . ***** Enfermeira - Professor Assistente do Departamento de Enfermagem da Universidade Federal do Rio Grande do Norte .
A análise esteve baseada num quadro referencial explicativo das relações existentes entre o ensino e a prática da assistência, no qual o ensino é conceituado como um dos elementos que determinam o estado da assistência da enfermagem em nossa instituição. Portanto, tenta-se descobrir qual a situação desse conjunto formado pelo ensino e a prática da assistência de enfermagem em nosso meio.

Os objetivos específicos foram de identificar os seguintes elementos do ensino teórico-prático no curso:

- a conceituação da assistência de enfermagem;

- as abordagens de ensino teórico-prático da assistência de enfermagem desenvolvidos pelos docentes;

- os critérios de avaliação da assistência de en . fermagem no ensino teórico-prático.

Através deste estudo espera-se contribuir para uma discussão mais ampla da categoria quanto a avaliação do cotidiano da assistência de enfermagem e as perspectivas de melhoria dessa assistência.

\section{METODOLOGIA}

A metodologia do estudo surgiu durante as pri- 
meiras discussões sobre o propósito abordado. Constatou-se que para realizar uma avaliação do cotidiano da assistência de enfermagem em nosso meio, sob o ponto de vista do ensino, era necessário primeiro descrever as vivências dos profissionais no. ensino da assistência, discuti-las, refleti-las e finalmente apresentar como é que estamos desenvolvendo esse ensino.

Daí, o desenvolvimento do estudo ter sido dividido em três fases. A primeira fase contituiu-se de uma discussão entre os enfermeiros docentes representantes das disciplinas, sobre o ensino teórico-prático da assistência de enfermagem que cada um estava desenvolvendo.* Questionou-se qual referencial teórico o professor utilizava no ensino da assistência, de que forma o conteúdo de ensino da assistência acompanhava a disciplina, e como esse conteúdo estava associado à prática assistencial de enfermagem.

$\mathrm{Na}$ segunda fase, cada docente representante voltou à disciplina que leciona e levou os mesmos questionamentos aos docentes de sua equipe. Na terceira fase, o grupo de estudo deu seguimento à análise a partir dos resultados das discussões nas disciplinas.

\section{O CURSO DE ENFERMAGEM DA UFRN}

O curso de graduação em Enfermagem e Obstetrícia da Universidade Federal do Rio Grande do Norte, foi criado pela Resolução no 58/73 - CONSU NI de 13/08/73, ingressando a sua primeira turma de 30 alunos em 1974. Foi reconhecido posteriormente através do Decreto no 82.026 de 24/07/78.

O ciclo básico é operacionalizado em três períodos com 25 disciplinas, perfazendo um total de 67 créditos a 1.006 horas aulas teóricas e teórico-práticas.

O ciclo profissional é organizado por área de atuação e operacionalizado em cinco períodos letivos, com 12 disciplinas, perfazendo um total de 80 créditos e 2.220 horas aulas teóricas e teórico-práticas e de estágios. Todas ministradas pelo Departamento de Enfermagem.

Desde a sua criação sof reu duas reformas curriculares, sendo que o currículo pleno em vigor atualmente, teve sua implantação em 1980 e se encontra em processo de estudo para reformulação.

Não tendo um marco conceitual explícito, o currículo é implicitamente guiado pelos objetivos do curso e pelo perfil profissional do enfermeiro que o curso propõe-se a formar. O perfil profissional foi elaborado e aprovado pelo colegiado de curso em 07/07/83 (UFRN, 1983) ${ }^{1}$. Ele apresenta as seguintes atribuições com relação à assistência para o enfermeiro graduado do curso de enfermagem da UFRN:

- Prestar assistência de enfermagem fundamentada no procedimento científico e sistemático da ciência da enfermagem;

- Demonstrar habilidade e destreza nas técnicas e procedimentos básicos de enfermagem, procurando desenvolver estudos e pesquisa visando uma tecnologia de enfermagem mais adequada à realidade;

- Planejar, organizar, coordenar, executar e avaliar a assistência de enfermagem. (p. 1)

As aulas práticas supervisionadas e/ou estágios são of erecidos em laboratórios ou serviços de atendi- mento à clientela quer se ja a nível individual, quer coletivo, através de serviços próprios da UFRN ou conveniados.

\section{O CONCEITO DA ASSISTÊNCIA DE ENFERMAGEM}

Trabalhando sob a premissa de que uma conceituação da assistência de enfermagem, definida e exposta, ajuda na compreensão dos objetivos do ensino quanto à prática da assistência, procurou-se caracterizar tal conceituação no curso. As discussões nesta fase tiveram como base os conceitos da assistência de enfermagem utilizados em cada disciplina.

Dentre as conceituações expostas pelas disciplinas, ficou evidenciado que persistiam alguns elementos em comum, tais como: a definição da assistência de enfermagem, como sendo, em essência, as ações sistematizadas, e a visão do sujeito dessa assistência como sendo o Homem, isto é, o indivíduo, família e comunidade no processo saúde/doença. As divergências centraram-se nos objetivos das ações de enfermagem. Enquanto que, para algumas disciplinas, o objetivo das ações era atender às necessidades humanas básicas no processo saúde/doença; para outras, a assistência de enfermagem objetiva a promoção, preservação e recuperação da saúde; ainda, para outras, o objetivo da assistência centra-se na melhoria da saúde da população ou na intervenção do processo saúde/doença.

A primeira vista, poderíamos afirmar que as ações sistematizadas servem de elo conceitual entre as disciplinas, pelo fato de as definições expostas apresentarem tal unidade. Porém, o que se observou nas discussões mais profundas sobre o significado do termo "ações sistematizadas", é que tais ações são conceituadas e implementadas de forma diferente nas discip inas. Em geral, a sistematização é adaptada à realidade de ensino e prática de cada disciplina, dando origem a diversas articulações sobre a essência dessa sistematização. Na prática docente, tal sistematização se desenvolve de maneira isolada pelos professores sem articulação com outras disciplinas e, muitas vezes, sem unidade dentro de uma mesma área de conhecimento.

Da mesma forma, as abordagens teóricas constituem um foco de divergências. Enquanto umas disciplinas enfatizam uma ou outra teoria de enfermagem, ou uma fundamentação teórica com origem em outra área de conhecimento; outras disciplinas não tem definida a base teórica de assistência de enfermagem utilizada no ensino de seu conteúdo. Neste último caso, o ensino se dá sem enfoque teórico, embora o seu conteúdo refleita a orientação teórica que direciona a assistência. A base teórica é passada para o aluno de forma isolada, e em algumas situaçōes e por alguns prof essores que ressaltam os aspectos científico e teorico, tornando-se, assim, uma abordagem desintegrada pela falta de continuidade e de aceitação até dentro da própria disciplina.

Toda esta problemática se reflete na descontinuidade de uma assistência de enfermagem não planejada e dicotomizada e, pela dissociação entre a teoria e prática, sentida pelos docentes e discentes no curso de graduação.

\footnotetext{
* As autoras agradecem às professoras Guiomar P. Barreto, Rita Ribeiro, Jussara Guerra, Oscarina Saraiva Coêlho e Suzana Maria Segundo Miranda pela colaboração dada no decorrer das discussōes deste trabalho.
} 
A questão de até que ponto tais diversificações contribuem para a insegurança do aluno, quanto ao que se espera dele na assistência de enfermagem de campo, seria um ponto para investigação. Contudo, pode-se supor que existe correlação, uma vez qué o aluno encontra-se na fase de aprendizagem na qual se estabelece uma base conceitual da sua atuação como enfermeiro.

Partindo do pressuposto de que a metodologia da assistência de enfermagem tem valor para o ensino e para a prática, faz-se necessário esclarecer que as ações sistematizadas são de utilidade na prática da assistência só quando estas se tornam viáveis nas reali dades existentes. Existe uma diferença entre ações sistematizadas, utilizadas como fim em si mesmas, e que enfatizam o detalhamento e as técnicas burocráticas, e aquelas ações sistematizadas com base científica e fundamentaç à teórica, utilizadas para viabilizar o desenvolvimento do processo natural e espontâneo de enfermagem. Como afirma PAIM (1979) ${ }^{2}$.

"O interesse em sistematicamente tratar o processo de enfermagem, chegou a dois pontos extremos. De um lado um grupo que exageradamente valoriza o método em detrimento da essência do processo de enfermagem tal como ele está aí, naquele momento da abordagem. Os que assim fazem em nome de uma busca cientifica desgastam termos e fabricam detalhamentos teóricos para as ações de enfermagem tornando-se por vezes uma tecnocrata, e perdendo a grande oportunidade de obter o reconhecimento pela competência demonstrada na simplicidade do fazer pelo pensar." (p. 67)

Questiona-se se as ações sistematizadas, entendida com a definição dos passos da metodologia da assistência da enfermagem, ou seja, a sistematização do processo de enfermagem, deverão ser eliminados em razão da inviabilidade verificada, haja vista as distorções sof ridas na sua implementação e, por razão das críticas, muitas vezes justificadas. Como aponta PAIM $(1979)^{3}$

"a simples recusa de uma abordagem científica representa grave retrocesso no progresso da ciência e dos valores humanos em enfermagem. As dificuldades de implantação de uma metodologia qualquer para abordagem do processo de enfermagem precisa significar o desafio suficiente aos estudos para adaptação, substituição, sem desvalorização da proposta teórica inicial." (p.68)

Frente às divergências e lacunas no que se refere às conceituações que norteam o ensino da assistência de enfermagem no curso, faz-se necessário um posi cionamento dos docentes em relação ao ensino dessa assistência. Paralelamente, sente-se a mesma necessidade em relação à sistematização das ações de enfermagem, como parte integral dessa assistência. Sentiu-se também a necessidade da elaboração de estudos mais profundos que indiquem uma forma adequada de visualizar e implementar as ações sistematizadas, para atingir os propósitos do curso e da assistência qualitativa.

\section{O ENSINO DA ASSISTÊNCIA DE ENFERMAGEM}

As deficiências e as desvinculações no ensino teórico prático de enfermagem, no Brasil, têm sido estudadas, analisadas e documentadas por vários autores, quer para promover a avaliação das práticas de ensino (PAM, 1982) ${ }^{3}$, quer com o propósito de despertar para uma questão mais social em busca da qualidade da assistência e do ensino (GERMANO, 1983; NAK AMAE, 1987) ${ }^{5,6}$

Assim, ao estudar como e de que forma se está ministrando esse ensino de assistência de enfermagem em nosso meio, faz-se necessário definirmos as abordagens utilizadas e a problemática da implantação das conceituações expostas.

Quanto às abordagens utilizadas, articulam-se vários processos metodológicos para o ensino de assistência de enfermagem dentro do curso. Umas disciplinas utilizam a metodologia de assistência como processo científico dessa assistência. Outras disciplinas utilizam assistência de enfermagem plane jada como sinônimo de assistência de enfermagem sistematizada. Abordagens baseadas nos conteúdos específicos orientados para o cuidado à doença são também utilizados. Outras disciplinas utilizam algumas teorias tais como a das Necessidades Humanas Básicas e a do Auto-cuidado. Esta diversificação acontece a nível de equipes e até de disciplinas.

A metodologia sistematizada da assistência parece ser o meio pelo qual a assistência é ensinada. Porém, embora o grupo disciplinar defina esta metodologia da assistência de enfermagem como sendo o método que se adapta ao ensino, na maioria dos casos, professores de uma mesma disciplina não a utiliza no ensino.

Surge, então, um ensino desvinculado dos pressu postos articulados dentro das disciplinas, gerando sentimentos de frustração, incerteza e insegurança, quanto aos objetivos de ensino, em alguns professores.

Já na prática, onde supômos que o aluno deveria ter a oportunidade de vivenciar concomitantemente aquelas conceituações sobre a assistência de enfermagem, a realidade que se observa, na maioria das disciplinas, é que isto não acontece. Se falamos em desvinculação no ensino teórico, essa desvinculação se dá com maior freqüência entre o ensino e a prática, em relação à assistência. Os professores observam que existe uma descontinuidade na assistência de enfermagem sistematizada abordada pelos alunos na prática. Com algumas exceções, quando o aluno e o professor saem de campo, aquela assistência sistematizada deixa de ser implementada.

Então surge a dúvida se a aplicação das ações sistematizadas no ensino prático é simplesmente um exercício didático sem ligação com a realidade, em vez do que deveria ser, a aplicação de conhecimentos científicos de enfermagem em situações concretas. É como se aquela abordagem sistematizada só servisse para propósitos de ensino. As críticas sentidas pelos docentes, oriundas dos enfermeiros de campo, baseam-se na complexibilidade de documentação, na dificuldade de operacionalizá-la sob condições de trabalho que não permitem uma assistência individualizada, e no tempo investido na sua implementação. Questiona-se, então, com que base as conceituações utilizadas no ensino são tomadas, e se, em algum momento, essas abordagens são elaboradas em acordo com os profissionais do serviço.

O grupo apontou alguns fatores que contribuem para a desvinculação no ensino teórico-prático, que foram: a desarticulação entre ensino e serviço quanto 
aos objetivos de enfermagem, e a desfamiliarização com o campo de prática pelos professores de enfermagem. Enfatizou-se este último porque sentiu-se que algumas soluções poderiam ser abordadas no sentido de promover maior atuação dos prof essores.

Se reconhecemos que o enfermeiro docente representa um profissional com maior e mais fácil acesso aos novos conhecimentos de enfermagem, em virtude da sua posição nos ambientes acadêmicos, nada mais lógico que sua contribuição no campo de prática ser bastante significativa. Porém, tal atuação não signficaria uma visita periódica para conhecer o campo de prática antes do estágio, como normalmente ocorre. Esta atuação iria, além do conceito docente-assistencial que enfatiza a manutenção do campo para recebimento do aluno. Seria uma atuação comprometida com o serviço, com horário e responsabilidades, em que se daria uma contribuição para a melhoria da qualidade de assistência com ou sem alunos presentes.

Em contrapartida, um comprometimento igualitário seria assumido pelos enfermeiros do serviço no sentido de que a responsabilidade permaneceria com o enfermeiro e que o trabalho, junto ao professor assistencial, se daria de forma paralela, em conjunto, com a oportunidade para um relacionamento profissional de intercâmbio e de aprendizagem, evitando assim, utilizar o professor como um substituto. Acredita-se que tal atuação traria benefícios não só ao serviço, como também ao professor, no sentido de contribuir para uma satisfação maior quanto à sua competência na prática e à sua colaboração na promoção da qualidade da assistência de enfermagem.

A integração ensino e assistência é de suma importância quando se observa, através de estudos ou depoimentos, que a assistência prestada nestes termos é benéfica e é apreciada pelo paciente/cliente (SANTOS, et alii, 1987) ${ }^{7}$. A nível de ensino, evidenciam-se algumas tentativas de integração interdisciplinar e en tre equipes. Porém, tais esforços permanecem muito a nível de comparação de conteúdos, com o objetivo de evitar duplicidades. Sentiu-se falta de tentativa de in tegração a nível de definição de conceituações da assistência de enfermagem, de elaboração de objetivos, e de como avaliar essa assistência.

Um outro aspecto, de ordem organizacional e que se apresenta como problema dentro do contexto do ensino teórico-prático, é a apropriação das unidades de conteúdo por parte dos professores.

A limitação do professor para uma área subespecializada em sua própria área de ensino, acarreta problemas de desintegração dentro da própria equipe, e até de distanciamento entre membros da mesma disciplina, uma vez que essa subespecialização tende a ser exclusiva.

Surge, então, o desconhecimento de certos as suntos por outros membros da equipe, acomodação para uma área de conhecimento do conteúdo, assim como a falta de comunicação entre membros da equipe, quanto à matéria e quanto às conceituações abordadas. Este fenômeno, algumas vezes, culmina na embaraçosa situação, em que o professor é reconhecido pela matéria específica que ensina, em vez de pelo próprio nome.

O problema de fragmentação das disciplinas, gerando apropriação de conteúdos, talvez reflita um problema maior de ordem curricular, visto que o curso em si está dividido em diferentes áreas de enfermagem. Se prestássemos atenção à mensagem que os nomes das disciplinas transmitem (Enfermagem Médico-Cirúrgico, Enfermagem Materno Infantil etc.), observaríamos que parece indicar que a prática e a assistência de enfermagem diferem entre disciplinas, dependendo da área de atuação na qual se dão.

\section{AVALIAÇÃO DA ASSISTÊNCIA DE ENFERMAGEM NO ENSINO}

Frente à contínua constatação de que a assistência de enfermagem se caracteriza cada vez mais como de má qualidade, as entidades de classe propõem a formulação de um modelo assistencial de enfermagem que contemple seu processo de trabalho e o atual momento de reorganização dos serviços de saúde (ABEn-Central, 1988) ${ }^{8}$.

Tal definição serviria para uma avaliação da qualidade. Definidos e discutidos os padrões de qualidade representariam o modelo daquilo que pretendemos que deva compor a assistência de enfermagem nas diversas situações.

Ao tentar identificar os padrões de qualidade uti lizados ao nível de ensino, para avaliação da assistência prestada no transcurso do ensino teórico-prático das disciplinas, o grupo chegou à conclusão de que tais parâmetros não existiam.

Constatou-se, ainda, que os parâmetros utilizados em algumas disciplinas, propostos por órgãos de saúde nacionais e internacionais, são mais uma listagem de atividades que descrevem a assistência, sem qualquer enfoque qualitativo da atividade a ser realizada. Devemos ressaltar, porém que algumas disciplinas conse guem dar uma abordagem mais qualitativa aos padrões utilizados na avaliação da prática do aluno no campo de estágio.

Contudo, o centro de avaliação dessas disciplinas, a nível da prática supervisionada, é o aluno; focalizando nos objetivos de aprendizagem, e na aplicação dos conceitos teóricos. Dá-se pouca ênfase à avaliação da qualidade da assistência prestada no processo do ensino teórico-prático e aos seus ef eitos ou repercussões na totalidade. Atualmente, porém, alguns docentes que implementam um programa docente-assistencial estão promovendo avaliação científica do programa para identificar o impacto de suas atividades sobre a saúde da população. Esse tipo de avaliação é de suma importância, pois, é através desse tipo de pesquisa que os padrões de assistência podem ser identificados e estabelecidos. Mas, isto é só um caso isolado. Precisamos de maiores esforços desta natureza.

Os padrões qualitativos e quantitativos da assistência dão origem aos padrões que servem como medidas do desempenho dos que fazem enfermagem. Portanto, a avaliação da assistência de enfermagem e dos profissionais que a praticam, deveria ser uma avaliação baseała em princípios administrativos e técnico-cien tíficos de grande utilidade para a organização de servigos (RIBEIRO, 1977) ${ }^{9}$.

Numa tentativa de apontar algumas considerações que poderiam servir de base para uma discussão mais profunda sc:tre o mcidelo da assistência, foram apontados alguns conceitos que poderiam qualificar tal assistência, como: continuidade, envolvimento com a totalidade e compromisso profissional incluíndo, neste 
último, competência técnica e conhecimento institucional.

\section{CONCLUSÃO}

O trabalho, ora apresentado, é um primeiro esforço para uma análise maior das tendências do ensino teórico-prático da assistência em enfermagem no curso de graduação em enfermagem da UFRN. Algumas considerações sobre a problemática enf rentada no ensino da assistência de enfermagem em geral foram levantadas e poderiam dar início a um debate sobre o assunto.

Sem aludirmos de que tudo está muito bem, expômos as nossas considerações quanto ao nosso ensino, assumindo uma postura de reflexão e autocrítica, tentando mostrar a necessidade de uma mudança direcionada para uma prática de ensino mais compatível e mais comprometida com as reais necessidades de saúde da população.

Concluíndo, reconhece-se a existência de uma problemática complexa no ensino da assistência de enfermagem no curso de enfermagem da UFRN que se caracteriza principalmente pela desintegração do ensino teórico-prático, especificamente refletindo:

- desarticulações internas quanto aos conceitos da assistência de enf ermagem ensinados;

- desvinculação do ensino na prática da assistência de enfermagem; e

- falta de padrões de qualidade para avaliação.

Tal conclusão, indica a necessidade de uma definição da base conceitual e teórica do exercício da assistência de enfermagem conjunto entre os enfermeiros docente e assistencial, e a nível de currículo do curso, para que ela possa direcionar a formação do enfermeiro para atuação na realidade existente.

Gostaríamos ainda de ressaltar que, no transcorrer deste trabalho, os participantes do grupo tiveram oportunidade de vivenciar, junto às disciplinas, um certo despertar para a necessidade de repensar as práticas de ensino, e de continuar o processo de discussões dentro das disciplinas.

Baseado nos resultados do estudo, o grupo propõe algumas sugestões que poderiam ser implementadas, para uma melhor especificação sobre o ensino e a prática da assistência de enfermagem no contexto do Rio Grande do Norte, a seguir:

- que os enfermeiros docente e assistencial realizem programações de discussões, seminários, oficinas de trabalho etc., que visem à elaboração de políticas institucionais quanto à melhoria da assistência de enfermagem e quanto ao trabalho integrado do ensino e serviço; .

- que enfermeiros docentes e de serviço, em conjunto. realizem estudos científicos sobre a definição e a qualidade da assistência de enfermagem, visando a elaboraçao de um modelo assistencial de enfermagem;
- que as disciplinas plane jem, elaborem e executem o programa de ensino teórico-prático numa ação conjunta entre enfermeiros docente e assistencial;

- que os docentes participem ativamente na reformulaçao do currículo do Curso de Enfermagem e na elaboração do seu marco conceitual.

\section{REFERÊNCIAS BIBLIOGRÁFICAS}

1 UNIVERSIDADE FEDERAL DO RIO GRANDE DO NORTE - URFN. Perfil Profissional, apostilha, julho, 1983.

2 PAIM, Lygia. Questões e mitos acerca de modelos de assistência de enfermagem. In: XXXI CONGRESSO BRASILEIRO DE ENFERMAGEM, Fortaleza, Ceará, 1979. Anais ... Fortaleza, Ceará, ABEn, 1979, pp. 61-9.

3 op. cit., p. 68

4 PAIM, Lygia, et alii. Objetivos do ensino de graduação em enfermagem. Revista Brasileira de Enfermagem, 1972.

5 GERMANO, Raimunda de Medeiros. Educação e Ideologia da Enfermagem no Brasil. São Paulo, Cortez, 1983. 118 p.

6 NAKAMAE, Djair Daniel. Novos Caminhos da Enfermagem. São Paulo, Cortez, 1987. 120 p.

7 SANTOS, Leila C. R. dos, et alii. O ensino da metodologia de assistência de enfermagem: responsabilidade da disciplina de fundamentos de enfermagem. Revista da Escola de Enfermagem da USP, São Paulo, 21(1): 75-88, abr. 1987.

8 ASSOCIAÇĀO BRASILEIRA DE ENFERMAGEM ABEn. Relatório de conclusōes recomendaçōes e moçōes do XL Congresso Brasileiro de Enfermagem. Belém, Pará, dez/1988. 20 p.

9 RIBEIRO, Circe de Melo. Avaliação da assistência de enfermagem. In: XXIX CONGRESSO BRASILEIRO DE ENFERMAGEM, Camboriú, Santa Catarina, 1977. Anais ... Camboriú, Santa Catarina, ABEn, 1977, pp 51-57.

\section{BIBLIOGRAFIA}

ALMEIDA, Maria Cecília Puntel de \& ROCHA, Juan Stuardo Y. O saber de Enfermagem e Sua Dimensão Prática. São Paulo, Cortez, 1986. 128 p.

CARVALHO, Judith Feitosa de. Modelo Operacional de Enfermagem com Enfoque Preventivo. Brasília, jul/83, mimeo, s.d.

CASTELLANOS, Brigitta E. P. Algumas reflexões sobre nossa profissão: a enfermagem. Revista Palista de Enfermagem, São Paulo, 2(2), nov/dez, 1982.

MARTINS, Ana Adelaide. A enfermagem como prática social. Revista Brasileira de Enfermagem. Brasília, 40(2/3) abr/maio/jun/jul/ago/set, 1987.

PAULA, Nara Sena de. A formação do enfermeiro. Revista Paulista de Enfermagem. São Paulo, 4(1), jan/fev/mar, 1984.

SILVA, Graciette Broges da. Enfermagem profissional: análise critica. São Paulo, Cortez, 1986. 143 p. 